\title{
The 2019 World Sleep Congress
}

Monica Levy Andersen ${ }^{1}$

${ }^{1}$ Universidade Federal de São Paulo, Departamento de Psicobiologia Rua Napoleão de Barros, 925, 04024002, São Paulo SP, Brazil.
Corresponding author: Monica Levy Andersen. E-mail: ml.andersen12@gmail.com
The $2^{\text {nd }}$ World Sleep Congress since the creation of the World Sleep Society was held in Vancouver, Canada from $20^{\text {th }}$ to $25^{\text {th }}$ September 2019. The Congress presented a wide range of clinical and pre-clinical findings in the field of Sleep Medicine. The most recent hot topics were presented in 89 symposia, 192 oral abstract presentations and 1,121 poster abstracts. We were also very pleased with the increasing internationalization of the Congress, with the presence of 3,583 attendees from 77 different countries. There was also a significant contribution made by Latin America countries. Prof. Dr. Dalva Poyares, from Brazil, a member of the World Sleep Society's International Sleep Research Training Program (ISRTP) Steering Committee, was also a member of the 2019 Program Committee of the Congress. Moreover, more than 90 researchers from Latin America (Brazil, Cuba, Chile, Colombia, Peru, Argentina, Mexico and Uruguay) attended the congress, being responsible for the presentation of more than 100 posters. Among the presented highlights, Prof. Dr. Luciano Drager made an important contribution on the link between obstructive sleep apnea (OSA), diurnal consequences and comorbidities. It is widely known that OSA leads to excessive daytime sleepiness, however, there is a lack of studies investigating how OSA is related to nighttime sleep duration, and vice-versa. In addition, environmental factors should also be considered, especially knowing that our current society is characterized by poor sleep. Other topics intensively explored were related to genetics, sleep hygiene and education, as well as the management of sleep disorders. The next World Sleep Congress will be held in Rio de Janeiro, Brazil, in 2021, and we are all very much looking forward to it, and to the opportunity to welcome our colleagues from around the world.

Monica Levy Andersen 